\title{
Extensions of the Mehler-Weisner and Other Results for the Hermite Function
}

\author{
By M. E. Cohen
}

\begin{abstract}
The purpose of this paper is to present expansions which generalize some well-known formulae for the Hermite function. Among these are the Weisner [20] extension of Mehler's [17] bilinear relation, some recent results of Carlitz [4], and the Bateman [2] addition theorem. A bilateral generating function involving the product of the Hermite and ultraspherical polynomials is given. Finally, some general polynomial expansion theorems are derived.
\end{abstract}

I. Introduction. Recently we proved the expansion [5]

$$
\begin{aligned}
& \sum_{n=0}^{\infty} \frac{(n+l) !(\alpha+\beta+l+1)_{n} t^{n}}{n !(\alpha+\beta+2 l+2)_{n}} P_{n+l}^{(\alpha, \beta)}(x) \\
& \quad=\frac{(2) l ! \Gamma(2 l+\alpha+\beta+2)}{\Gamma(l+\alpha+1) \Gamma(l+\beta+1) t^{\alpha+\beta+l+1}} P_{l}^{(\alpha, \beta)}\left(\frac{1-\rho}{t}\right) Q_{l}^{(\alpha, \beta)}\left(\frac{1+\rho}{t}\right),
\end{aligned}
$$

where $\rho=\left(1-2 x t+t^{2}\right)^{1 / 2},|t|<1, l$ is a nonnegative integer, and $P$ and $Q$ are the Jacobi polynomial and function, respectively. This type of generating function, where the degree of the polynomial is $(n+l)$, was previously not known for the Jacobi polynomial. The result (1.1) prompted us to investigate expansions of this type for bilinear generating functions.

For the Hermite polynomial, Mehler gave

$$
\sum_{n=0}^{\infty} \frac{t^{n}}{2^{n} n !} H_{n}(x) H_{n}(y)=\left(1-t^{2}\right)^{-1 / 2} \exp \left[y^{2}-\frac{(y-x t)^{2}}{1-t^{2}}\right] .
$$

See also Askey [1, p. 16, Eq. 2.44w], [1,p. 35]. Erdélyi [7], Feldheim [8], Hardy [12], Hille [13], Sarmanov et al. [18], Watson [19], and others have worked on (1.2). A hundred years later Weisner [20], with the aid of group theoretical methods, generalized (1.2) to give

$$
\begin{aligned}
& \sum_{n=0}^{\infty} \frac{t^{n}}{2^{n} n !} H_{n}(x) H_{n+\nu}(y) \\
& \quad=\left(1-t^{2}\right)^{-1 / 2 \nu-1 / 2} \exp \left[\frac{y^{2} t^{2}-2 x y t+t^{2} x^{2}}{t^{2}-1}\right] \cdot H_{\nu}\left[\frac{y-x t^{\circ}}{\left(1-t^{2}\right)^{1 / 2}}\right],
\end{aligned}
$$

where $|t|<1$, and $\nu$ is an arbitrary constant. $H_{\nu}$ is the Hermite function, defined as

Received August 7, 1975; revised September 30, 1975.

AMS (MOS) subject classifications (1970). Primary 42A56, 42A60; Secondary 33A65.

Key words and phrases. Addition theorem, bilinear generating function, Gegenbauer polynomial, generalized function, Hermite function.

Copyright @ 1976, American Mathematical Society 


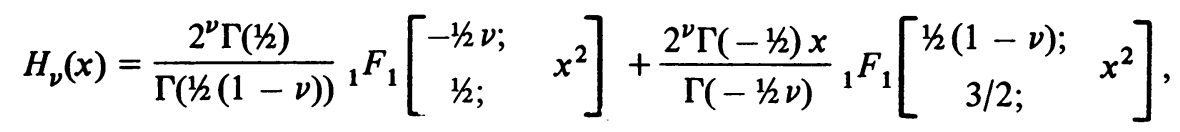

valid for all complex values of $x$ and $\nu$, and $\Gamma(\nu)$ is the gamma function. (1.3) is not extensively quoted in the literature and it was not known to the author when this work was done. Among other results in his paper, Carlitz [4] reproves (1.3) using series iteration and integration, but for the case $\nu$ a nonnegative integer.

We now present one generalization of (1.3).

$$
\sum_{n=0}^{\infty} \frac{t^{n}}{n !} G_{n, s}(y, \alpha) H_{n, s}(x, \lambda)=\left(1+\lambda t^{s}\right)^{\alpha-1} G_{0, s}\left[\frac{y-x t}{\left(1+\lambda t^{s}\right)^{1 / s}}, \alpha\right],
$$

$\left|\lambda t^{s}\right|<1$, where

$$
G_{n, s}(y, \alpha)=\sum_{p=0}^{\infty} \frac{(-1)^{p} y^{p}}{p ! \Gamma(\alpha-n / s-p / s)}
$$

is valid for complex values of $\alpha, n$, and $y$, for $s$ an integer $\geqslant 2$.

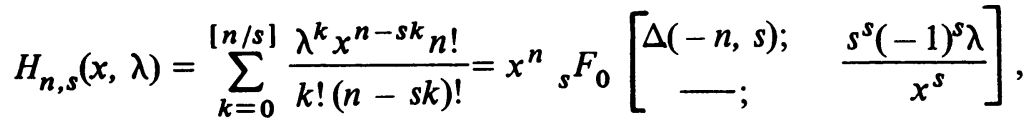

where $\Delta(-n, s)=-n / s,(-n+1) / s, \ldots,(-n+s-1) / s$. Note that the Hermite function defined in (1.4) is connected with (1.6) through the relation

$$
G_{n, 2}(2 x, 1 / 2-1 / 2 v)=\frac{2^{-n-\nu} \exp \left(-x^{2}\right)}{\pi^{1 / 2}} H_{n+\nu}(x)
$$

and with the generalized Hermite polynomial (1.7) by

$$
H_{n, 2}(2 x,-1)=H_{n}(x) .
$$

One could consider (1.6) as a definition for a generalized Hermite function. On the other hand, (1.7) is the well-known generalized Hermite polynomial, which occurs in statistical problems. See Gupta and Jain [11], Gould and Hopper [9] , and also Lukacs [15] for properties and applications. In Cohen [6], expansion problems connected with this polynomial are established. Putting $s=2, \alpha=1 / 2-1 / 2 \nu, \lambda=-1$ in (1.5), and algebraic manipulation yields Weisner's result (1.3).

(1.5) is in fact a special case of the more general expansion

$$
\begin{aligned}
& \sum_{n_{1}, n_{2}, \ldots, n_{i}} G_{N, s}(y, \mu) \prod_{j=1}^{i} \frac{t_{j}^{n_{j}}}{n_{j} !} H_{n_{j}, s}\left(x_{j}, \lambda_{j}\right) \\
& =\left\{1+\sum \lambda_{j} t_{j}^{s}\right\}^{\mu-1} G_{0, s}\left[\frac{y-\sum t_{j} x_{j}}{\left\{1+\sum \lambda_{j} t_{j}^{s}\right\}^{1 / s}}, \mu\right]
\end{aligned}
$$

for $\left|\Sigma \lambda_{j} t_{j}^{s}\right|<1$, where $N=n_{1}+n_{2}+\cdots+n_{i}$, and

$$
\sum x_{j} t_{j}=x_{1} t_{1}+x_{2} t_{2}+\cdots+x_{i} t_{i}, \quad \sum \lambda_{j} t_{j}^{s}=\lambda_{1} t_{1}^{s}+\lambda_{2} t_{2}^{s}+\cdots+\lambda_{i} t_{i}^{s} .
$$


In (1.10), let $s=2, y=2 \eta, \mu=1 / 2-1 / 2 v, \lambda_{j}=-1, x_{j}=2 \xi_{j}$. Then for the Hermite polynomial

$$
\begin{aligned}
& \sum_{n_{1}, n_{2}, \ldots, n_{i}} 2^{-N} H_{N+\nu}(\eta) \prod_{j=1}^{i} \frac{t_{j}^{n_{j}}}{n_{j} !} H_{n_{j}}\left(\xi_{j}\right) \\
& \quad=\exp \left[\eta^{2}-\frac{\left(\eta-\sum t_{j} \xi_{j}\right)^{2}}{\left(1-\sum t_{j}^{2}\right)}\right]\left\{1-\sum t_{j}^{2}\right\}^{-1 / 2-1 / 2 \nu} H_{\nu}\left[\frac{\eta-\sum t_{j} \xi_{j}}{\left(1-\sum t_{j}^{2}\right)^{1 / 2}}\right]
\end{aligned}
$$

For $\nu=0$, one obtains Eq. (2.3) of Carlitz [4], which is one of the main results of his paper. function

Now Bateman [2, p. 890, Eq. 29] derived an addition theorem for the Hermite

$$
\left(1+t^{2}\right)^{1 / 2 \nu} H_{\nu}\left[\frac{y+x t}{\left(1+t^{2}\right)^{1 / 2}}\right]=\sum_{n=0}^{\infty} \frac{(-1)^{n}(-v)_{n} t^{n} H_{\nu-n}(y) H_{n}(x)}{n !}
$$

where $\left(\begin{array}{l}\nu \\ n\end{array}\right)=(-1)^{n}(-\nu)_{n} / n !$ and $|t|<1$.

For the case $\nu$ a nonnegative integer, (1.12) was first given by Kampé de Fériet [14]. (1.12) is also essentially an addition theorem for the parabolic cylinder function as

$$
H_{\nu}(x)=2^{1 / 2 \nu} \exp \left(1 / 2 x^{2}\right) D_{\nu}\left(2^{1 / 2} x\right) \quad[16, \text { p. } 212, \text { Eq. 5] }
$$

We present a generalization of (1.12):

$$
\sum_{n=0}^{\infty} \frac{t^{n}}{n !} \bar{G}_{n, s}(y, \alpha) H_{n, s}(x, \lambda)=\left(1-\lambda t^{s}\right)^{-\alpha} \bar{G}_{0, s}\left[\frac{y-x t}{\left(1-\lambda t^{s}\right)^{1 / s}}, \alpha\right]
$$

for $\left|\lambda t^{s}\right|<1$, where

$$
\bar{G}_{n, s}(y, \alpha)=\sum_{p=0}^{\infty} \frac{(-1)^{p} y^{p} \Gamma(\alpha+n / s+p / s)}{p !}
$$

is valid for complex values of $\alpha, n$ and $y$, for $s$ an integer $\geqslant 2$. (1.14) is seen to be connected to the Hermite function through the relation

$$
\bar{G}_{n, 2}(2 y, 1 / 2 a)=2 \Gamma(n+a) H_{-a-n}(y) .
$$

Putting $s=2, \alpha=-1 / 2 v, \lambda=-1$ in (1.13) and simplifying gives (1.12). We have, in fact, a more general expression of which (1.13) is a particular example.

$$
\begin{aligned}
\sum_{n_{1}, n_{2}, \ldots, n_{i}} \bar{G}_{N, s}(y, \mu) \prod_{j=1}^{i} \frac{t_{j}^{n_{j}}}{n_{j} !} H_{n_{j}, s}\left(x_{j}, \lambda_{j}\right) \\
\quad=\left\{1-\sum \lambda_{j} t_{j}^{s}\right\}^{-\mu} \bar{G}_{0, s}\left[\frac{y-\sum t_{j} x_{j}}{\left\{1-\sum \lambda_{j} t_{j}^{s}\right\}^{1 / s}}, \mu\right]
\end{aligned}
$$

for $\left|\Sigma \lambda_{j} t_{j}^{s}\right|<1$, where the symbols are defined in (1.10). Letting $y=2 \eta, s=2, \mu=$ 
$-1 / 2 v, \lambda_{j}=-1, x_{j}=2 \xi_{j}$ in (1.16), the following expression for the Hermite polynomial presents itself.

$$
\begin{array}{r}
\sum_{n_{1}, n_{2}, \ldots, n_{i}}(-\nu)_{N} H_{\nu-N}(\eta) \prod_{j=1}^{i} \frac{t_{j}^{n_{j}}}{n_{j} !} H_{n_{j}}\left(\xi_{j}\right) \\
=\left\{1+\sum t_{j}^{2}\right\}^{1 / 2 \nu} H_{\nu}\left[\frac{\eta-\sum t_{j} \xi_{j}}{\left\{1+\sum t_{j}^{2}\right\}^{1 / 2}}\right] .
\end{array}
$$

Another extension of (1.12) is derived for the case $\nu$ a nonnegative integer. This addition theorem for the generalized Hermite polynomial takes the finite form

$$
\sum_{n=0}^{l} \frac{(-l)_{n}}{n !} t^{n} H_{n, s}(x, \lambda) H_{l-n, s}(y, \mu)=H_{l, s}\left(y-x t, \mu+\lambda(-t)^{s}\right) .
$$

$H_{n, s}(x, \lambda)$ is defined in (1.7). For $s=2, \lambda=-1, x \rightarrow 2 x, y \rightarrow 2 y, \nu=l$, (1.18) reduces to (1.12), for $\nu$ a nonnegative integer. (1.18) admits a more general expression

$$
\begin{aligned}
\sum_{n_{1}, n_{2}, \ldots, n_{i}}^{N \leqslant l}(-l)_{N} H_{l-N, s}(y, \mu) \prod_{j=1}^{i} \frac{t_{j}^{n_{j}}}{n_{j} !} H_{n_{j}, s}\left(x_{j}, \lambda_{j}\right) \\
=H_{l, s}\left[y-\sum t_{j} x_{j}, \mu+(-1)^{s} \sum \lambda_{j} t_{j}^{s}\right]
\end{aligned}
$$

where $N=n_{1}+n_{2}+\cdots+n_{i}$.

The next result presented is the bilateral generating function involving the Hermite and Gegenbauer polynomials.

$$
\begin{aligned}
\sum_{n=0}^{\infty} \frac{(-1)^{n} t^{n}}{2^{n}(1 / 2 \nu+1 / 2-1 / 2 n)_{n} \Gamma(1 / 2-1 / 2 \nu-1 / 2 n)} C_{n}^{1 / 2 \nu+1 / 2-1 / 2 n}(x) H_{n}(y) \\
=\frac{2^{-\nu}\left(1+t^{2}\right)^{1 / 2 \nu}}{\pi^{1 / 2}}\left\{1+t^{2}\left(1-x^{2}\right)\right\}^{-1 / 2 \nu-1 / 2} \\
\cdot \exp \left[\frac{y^{2} t^{2}\left(1-x^{2}\right)}{1+t^{2}\left(1-x^{2}\right)}\right] H_{\nu}\left[\frac{x y t}{\left(1+t^{2}\right)^{1 / 2}\left(1+t^{2}-t^{2} x^{2}\right)^{1 / 2}}\right],
\end{aligned}
$$

where $\left|t^{2}\left(1-x^{2}\right)\right|<1$. For other bilateral relations pertaining to the Hermite polynomial, see Brafman [3].

The results in the introduction are proved in Section II. The method of proof differs from that in Carlitz [4], Hille [13], Watson [19], and others. Section III is devoted to the presentation and proof of expansions involving more generalized polynomials. These expressions appear to give new and known results for the Hermite and ultraspherical polynomials.

II. Proofs of Section I Results.

(i) Proof of (1.5) and (1.10). The general proof of (1.10) is given, which includes its one-dimensional special case (1.5). 
The left-hand side of (1.10) is by definition

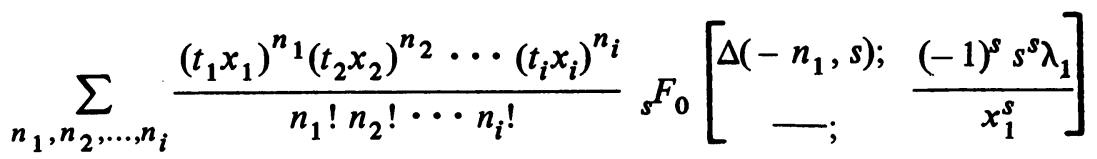

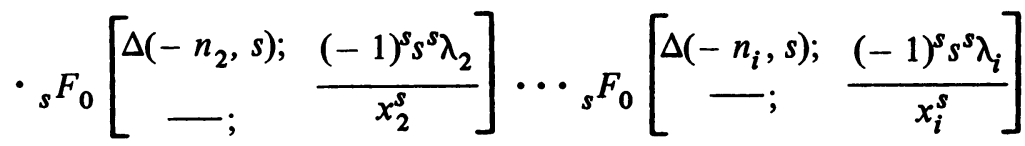

$$
\begin{aligned}
& \text { - } \sum_{p=0}^{\infty} \frac{(-1)^{p} y^{p}}{p ! \Gamma\left(\alpha-n_{1} / s-n_{2} / s-\cdots-n_{i} / s-p / s\right)} \text {. }
\end{aligned}
$$

Using the double series transformation

$$
\sum_{n=0}^{\infty} \sum_{k=0}^{[n / s]} f(n, k)=\sum_{n=0}^{\infty} \sum_{k=0}^{\infty} f(n+s k, k)
$$

for $i$ sums

$$
n_{1} \rightarrow n_{1}+s k_{1}, n_{2} \rightarrow n_{2}+s k_{2}, \ldots, n_{i} \rightarrow n_{i}+s k_{i},
$$

the gamma function relation

$$
\begin{aligned}
& 1 / \Gamma\left(\alpha-p / s-n_{1} / s-n_{2} / s-\cdots-n_{i} / s-k_{1}-k_{2}-\cdots-k_{i}\right) \\
& =\frac{(-1)^{k_{1}+k_{2}+\cdots+k_{i}\left(1-\alpha+p / s+n_{1} / s+n_{2} / s+\cdots+n_{i} / s\right)_{k_{1}}+k_{2}+\cdots+k_{i}},}{\Gamma\left(\alpha-p / s-n_{1} / s-n_{2} / s-\cdots-n_{i} / s\right)},
\end{aligned}
$$

and the expansion

$$
\begin{aligned}
\sum_{k_{1}, k_{2}, \cdots, k_{i}} \frac{\left(1-\alpha+p / s+n_{1} / s+n_{2} / s+\cdots+n_{i} / s\right)_{k_{1}+k_{2}+\cdots+k_{i}}}{k_{1} ! k_{2} ! \cdots k_{i} !} \\
\cdot\left(-\lambda_{1} t_{1}^{s}\right)^{k_{1}}\left(-\lambda_{2} t_{2}^{s}\right)^{k_{2}} \cdots\left(-\lambda_{i} t_{i}^{s}\right)^{k_{i}} \\
=\left(1+\lambda_{1} t_{1}^{s}+\lambda_{2} t_{2}^{s}+\cdots+\lambda_{i} t_{i}^{s}\right)^{\alpha-1-n_{1} / s-n_{2} / s-\cdots-n_{i} / s-p / s},
\end{aligned}
$$

(2.1) simplifies to

$$
\begin{aligned}
& \sum_{n_{1}, n_{2}, \ldots, n_{i}} \sum_{p=0}^{\infty} \frac{\left(x_{1} t_{1}\right)^{n_{1}}\left(x_{2} t_{2}\right)^{n_{2}} \cdots\left(x_{i} t_{i}\right)^{n_{i}}(-1)^{p} y^{p}}{n_{1} ! n_{2} ! \cdots n_{i} ! p ! \Gamma\left(\alpha-p / s-n_{1} / s-n_{2} / s-\cdots-n_{i} / s\right)} \\
& \cdot\left(1+\lambda_{1} t_{1}^{s}+\lambda_{2} t_{2}^{s}+\cdots+\lambda_{i} t_{i}^{s}\right)^{\alpha-1-n_{1} / s-n_{2} / s-\cdots-n_{i} / s-p / s} \\
&=\sum_{p=0}^{\infty} \sum_{n_{1}, \cdots, n_{i}}^{<p} \frac{(-1)^{p}(-p)_{n_{1}}+\cdots+n_{i}\left(x_{1} t_{1}\right)^{n_{1}} \cdots\left(x_{i} t_{i}\right)^{n_{i}}}{n_{1} ! \cdots n_{i} ! p ! \Gamma(\alpha-p / s)}
\end{aligned}
$$


(2.6)

$$
\begin{aligned}
y^{p-n_{1}-\cdots-n_{i}}\left(1+\lambda_{1} t_{1}^{s}+\cdots+\lambda_{i} t_{i}^{s}\right)^{\alpha-1-p / s} \\
=\sum_{p=0}^{\infty} \frac{(-1)^{p}\left(1+\lambda_{1} t_{1}^{s}+\cdots+\lambda_{i} t_{i}^{s}\right)^{\alpha-1-p / s}\left(y-t_{1} x_{1}-\cdots-t_{i} x_{i}\right)^{p}}{p ! \Gamma(\alpha-p / s)} .
\end{aligned}
$$

Going from (2.5) to (2.6) entails the use of the series transformation, and from (2.6) to (2.7) we have employed an expansion similar to (2.4).

(2.7) is the right-hand side of (1.10) by definition.

(ii) Proof of (1.13) and (1.16). Again, it is sufficient to prove (1.16).

We follow the procedure as in the proof of (1.10), with modifications.

The left-hand side of (1.16) may be expressed as

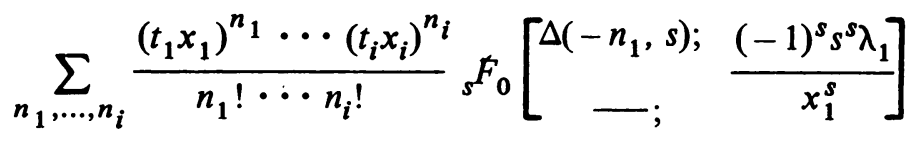

$$
\begin{aligned}
& \cdots{ }_{s} F_{0}\left[\begin{array}{ll}
\Delta\left(-n_{i}, s\right) ; & \left.\frac{(-1)^{s} s^{s} \lambda_{i}}{x_{i}^{s}}\right]
\end{array}\right. \\
& \cdot \sum_{p=0}^{\infty} \frac{(-1)^{p} y^{p} \Gamma\left(\alpha+n_{1} / s+\cdots+n_{i} / s+p / s\right)}{p !} \text {. }
\end{aligned}
$$

Using the double series transformation (2.2), the gamma function simplification

$$
\begin{aligned}
\Gamma(\alpha+ & \left.n_{1} / s+\cdots+n_{i} / s+p / s+K\right) \\
& =\Gamma\left(\alpha+n_{1} / s+\cdots+n_{i} / s+p / s\right)\left(\alpha+n_{1} / s+\cdots+n_{i} / s+p / s\right)_{K}
\end{aligned}
$$

where $K=k_{1}+\cdots+k_{i}$, the remainder of the proof is essentially the same as in (i), giving the right-hand side of (1.16).

(iii) Proof of (1.18) and (1.19). It suffices to prove (1.19).

The left-hand side of (1.19) can be put in the form

$$
\begin{aligned}
& \sum_{n_{1}, \cdots, n_{i}}^{N \leqslant l} \frac{(-l)_{N}\left(t_{1} x_{1}\right)^{n_{1}} \cdots\left(t_{i} x_{i}\right)^{n_{i}} y^{l-N}}{n_{1} ! \cdots n_{i} !}{ }_{s} F_{0}\left[\begin{array}{cc}
\Delta\left(-n_{1}, s\right) ; & \left.\frac{(-1)^{s} s^{s} \lambda_{1}}{x_{1}^{s}}\right] \\
-; & ;
\end{array}\right. \\
& \ldots{ }_{s} F_{0}\left[\begin{array}{cc}
\Delta\left(-n_{i}, s\right) ; & \frac{(-1)^{s} s^{s} \lambda_{i}}{x_{i}^{s}}
\end{array}\right]{ }_{s} F_{0}\left[\begin{array}{cc}
\Delta(-l+N) / s ; & \frac{(-1)^{s} \mu}{y^{s}}
\end{array}\right],
\end{aligned}
$$

where $N=n_{1}+\cdots+n_{i}$. With the aid of the double series transformation (2.2) for $i$ sums, $n_{i} \rightarrow n_{i}+s k_{i}$ and the expansion

$$
\begin{gathered}
\sum_{n_{1}, \cdots, n_{i}} \frac{\left(-l+s k_{1}+\cdots+s k_{i}+s p\right)_{N}}{n_{1} ! \cdots n_{i} !}\left(\frac{t_{1} x_{1}}{y}\right)^{n_{1}} \cdots\left(\frac{t_{i} x_{i}}{y}\right)^{n_{i}} \\
=\left\{1-\frac{t_{1} x_{1}}{y}-\cdots-\frac{t_{i} x_{i}}{y}\right\}^{l-s k_{1}-\cdots-s k_{i}-s p}
\end{gathered}
$$

(2.10) reduces to 


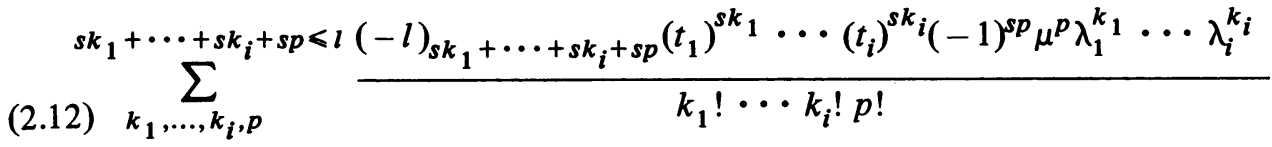

$$
\cdot\left(y-t_{1} x_{1}-\cdots-t_{i} x_{i}\right)^{l-s k_{1}-\cdots-s k_{i}-s p} .
$$

With the aid of the series iteration

$$
\sum_{k_{1}, \ldots, k_{i}, p} f\left(p, k_{1}, \ldots, k_{i}\right)=\sum_{p=0}^{\infty} \sum_{k_{1}, \ldots, k_{i}}^{K \leqslant p} f\left(p-K, k_{1}, \ldots, k_{i}\right)
$$

where $K=k_{1}+\cdots+k_{i}$, and

$$
\begin{gathered}
\sum_{k_{1}, \ldots, k_{i}}^{K \leqslant p} \frac{\mu^{p-K}(-p)_{K}(-1)^{(s+1) K} t_{1}^{s k_{1}} \cdots t_{i}^{s k_{i}} \lambda_{1}^{k_{1}} \cdots \lambda_{i}^{k_{i}}}{k_{1} ! \cdots k_{i} !} \\
=\left\{\mu+\lambda_{1}\left(-t_{1}\right)^{s}+\cdots+\lambda_{i}\left(-t_{i}\right)^{s}\right\}^{p},
\end{gathered}
$$

(2.12) simplifies to give

$$
\begin{gathered}
\left(y-t_{1} x_{1}-\cdots-t_{i} x_{i}\right)^{l} \sum_{p=0}^{[l / s]} \frac{\left(y-t_{1} x_{1}-\cdots-t_{i} x_{i}\right)^{-s p}(-1)^{s p}(-l)_{s p}}{p !} \\
\cdot\left\{\mu+\lambda_{1}\left(-t_{1}\right)^{s}+\cdots+\lambda_{i}\left(-t_{i}\right)^{s}\right\}^{p}
\end{gathered}
$$

(2.15) is the right-hand side of (1.19).

(iv) Proof of (1.20). The left-hand side of (1.20) is expressed as

$\sum_{n=0}^{\infty} \frac{(-x t)^{n}}{\Gamma(1 / 2-1 / 2 \nu-1 / 2 n)}{ }_{2} F_{1}\left[\begin{array}{c}-1 / 2 n,-1 / 2 n+1 / 2 ; \\ 1 / 2-1 / 2 v-1 / 2 n ;\end{array}\right] \sum_{k=0}^{[n / 2]} \frac{2^{n-2 k} y^{n-2 k}(-1)^{k}}{k !(n-2 k) !}$

$$
=\sum_{n=0}^{\infty} \sum_{k=0}^{[n / 2]} \frac{(-2 x y t)^{n}(-1)^{k}\left(1-1 / x^{2}\right)^{1 / 2 n-1 / 2 v}}{k ! 2^{k}(n-2 k) ! \Gamma(1 / 2-1 / 2 v-1 / 2 n) y^{2 k}}
$$

$$
\text { - }{ }_{2} F_{1}\left[\begin{array}{rr}
-1 / 2 \nu,-1 / 2 \nu+1 / 2 ; & 1 \\
1 / 2-1 / 2 \nu-1 / 2 n ; & x^{2}
\end{array}\right] \text {. }
$$

$$
\sum_{n, p, k=0}^{\infty} \frac{\left[-2 y t\left(x^{2}-1\right)^{1 / 2}\right]^{n}\left[\left(x^{2}-1\right) t^{2}\right]^{k}\left[\left(1-1 / x^{2}\right)\right]^{-1 / 2}(-1 / 2 \nu)_{p}(1 / 2-1 / 2 \nu)_{p}(1 / 2 \nu+1 / 2+1 / 2 n-p)_{k}}{n ! p ! k ! \Gamma(1 / 2-1 / 2 \nu-1 / 2 n+p) x^{2 p}} .
$$

In (2.16) we have used the representation (3.20) for the ultraspherical polynomial. (2.16) to (2.17) implies the Kummer transformation. (2.18) results from (2.17) by using the double series transformation (2.2).

Now

$$
\sum_{k=0}^{\infty} \frac{(1 / 2 v+1 / 2+1 / 2 n-p)_{k}}{k !}\left\{t^{2}\left(x^{2}-1\right)\right\}^{k}=\left[1+t^{2}\left(1-x^{2}\right)\right]^{-1 / 2 v-1 / 2-1 / 2 n+p}
$$

Combining (2.18) and (2.19) and using once again the Kummer transformation gives 


$$
\begin{aligned}
\sum_{n=0}^{\infty} \sum_{p=0}^{[n / 2]} \frac{(-2 x y t)^{n}\left[1+t^{2}\left(1-x^{2}\right)\right]^{-1 / 2 \nu-1 / 2-1 / 2 n}\left(1+t^{2}\right)^{1 / 2 \nu-1 / 2 n}}{2^{2 p} p !(n-2 p) ! \Gamma(1 / 2-1 / 2 \nu-1 / 2 n+p)} \\
\cdot\left[\frac{1+t^{2}\left(1-x^{2}\right)}{x^{2}}\right]^{p} \\
=\sum_{n=0}^{\infty} \frac{(-2 x y t)^{n}\left[1+t^{2}\left(1-x^{2}\right)\right]^{-1 / 2 \nu-1 / 2-1 / 2 n}\left(1+t^{2}\right)^{-1 / 2 n+1 / 2 \nu}}{n ! \Gamma(1 / 2-1 / 2 \nu-1 / 2 n)} \\
\cdot \sum_{p=0}^{\infty} \frac{\left[y^{2} t^{2} /\left(1+t^{2}\right)\right]^{p}}{p !} .
\end{aligned}
$$

From (2.20) to (2.21) we have used the double series transformation. In (2.21) the series over $p$ is seen to be the exponential, and using the definition of the Hermite function gives the required right-hand side of $(1.20)$.

III. Generalized Polynomial Expansions. This section deals with general expansion theorems. Some special cases are

$$
\text { (a) } \begin{aligned}
& \sum_{n=0}^{\infty} \frac{(n+l) !}{(2 \nu)_{n}} y^{n} C_{n}^{\nu}(x) C_{n+l}^{\mu}(x y) \\
& =\frac{(-1)^{1 / 2 l} l !(\mu)_{1 / 2 l}}{(1 / 2 l) !\left(1-x^{2} y^{2}\right)^{\mu+1 / 2 l}}{ }_{2} F_{1}\left[\begin{array}{c}
1 / 2 l+\mu, 1 / 2 l+1 / 2 ; \\
\nu+1 / 2 ;
\end{array} \quad \frac{y^{2}\left(1-x^{2}\right)}{\left(1-x^{2} y^{2}\right)}\right] \text { for } l \text { even, } \\
& =0 \text { for } l \text { odd }
\end{aligned}
$$

and $|y|<1$, where $C_{m}^{\lambda}(x)$ is the Gegenbauer polynomial. For special values, the righthand side of (3.1) may be expressed as well-known functions, including the Jacobi function. It also reduces to simple expressions for $2 \nu=l$ or $2 \nu-2 \mu+1=l$.

(b) $\sum_{n=0}^{\infty} \frac{(n+l) !}{2^{n} n !} y^{n} H_{n}(x) C_{n+l}^{\mu}(x y)$

$$
\begin{aligned}
& \cong(\mu)_{1 / 2 l}\left(1-x^{2} y^{2}\right)^{-\mu-1 / 2 l}{ }_{2} F_{0}\left[\begin{array}{c}
1 / 2 l+1 / 2,1 / 2 l+\mu ; \\
-;
\end{array}\right. \\
& =0 \text { for } l \text { odd, }
\end{aligned}
$$

where the right-hand side is a divergent series. See Luke [16, Vol. I, Chapter IV, Section (4.7), and Chapter VI, Sections (6.2.7) and (6.2.11)] for a fine exposition of the asymptotic expansion and significance of the ${ }_{2} F_{0}$ divergent series.

$$
\text { (c) } \begin{aligned}
\sum_{n=0}^{\infty} \frac{(x y)^{n}}{(l)_{n}} C_{n}^{1 / 2 l}(x) H_{n+l}(y) & =\frac{(-1)^{1 / 2 l} l !}{(1 / 2 l) !} \exp \left(\frac{y^{2}}{x^{2}}\right) \text { for } l \text { even, } \\
& =0 \quad \text { for } l \text { odd }
\end{aligned}
$$

where $|y|<1$. 
We now proceed with the proof of some general theorems.

For $\left\{a_{k}\right\}$ and $\left\{b_{k}\right\}$ arbitrary complex sequences, $\mu$ an arbitrary complex number, and $l$ a nonnegative integer,

$$
\begin{aligned}
\sum_{n=0}^{\infty} \frac{\xi^{n}}{n !} H_{n+l}(\xi) A_{n}(\eta) & =\frac{(-1)^{1 / 2 l} l !}{(1 / 2 l) !} \theta(\xi, \eta) & & \text { for } l \text { even, } \\
& =0 & & \text { for } l \text { odd }
\end{aligned}
$$

where $|\xi|<1$, and

$$
\begin{aligned}
& A_{n}(\eta)=\sum_{k=0}^{[n / 2]} \frac{(-1)^{k} \eta^{2 k}(-n)_{2 k}\left\{a_{k}\right\}}{2^{2 k} k !(1 / 2 l+1 / 2)_{k}} \\
& \theta(\xi, \eta)=\exp \left(\xi^{2}\right) \sum_{k=0}^{\infty} \frac{\left(\xi^{2} \eta^{2}\right)^{k}\left\{a_{k}\right\}}{k !} \\
& \sum_{n=0}^{\infty} \frac{(1 / 2 l)_{n} \xi^{n}}{(l)_{n} n !} D_{n}(\eta) B_{n+l}(\xi)=\frac{(-1)^{1 / 2 l} l !}{(1 / 2 l) !} \Phi(\xi, \eta) \quad \text { for } l \text { even } \\
& =0 \quad \text { for } l \text { odd, }
\end{aligned}
$$

where $|\xi|<1$, and

$$
\begin{gathered}
B_{n+l}(\xi)=\sum_{k=0}^{[1 / 2(n+l)]} \frac{[-(n+l)]_{2 k}(-1)^{k}\left\{a_{1 / 2 l+n-k}\right\} \xi^{n+l-2 k}}{k ! 2^{2 k-n-l}}, \\
D_{n}(\eta)=\sum_{p=0}^{[n / 2]} \frac{(-n)_{2 p}\left\{b_{p}\right\} \eta^{2 p}}{2^{2 p-n} p ! \cdot(1-1 / 2 l-n)_{p}}, \\
\Phi(\xi, \eta)=\sum_{k=0}^{\infty} \frac{\left\{a_{k}\right\}\left\{b_{k}\right\} \eta^{2 k} \xi^{2 k}}{k !},
\end{gathered}
$$

$$
\begin{aligned}
\sum_{n=0}^{\infty} \frac{(n+l) ! \xi^{n}}{n !} C_{n+l}^{\mu}(\xi) A_{n}(\eta) & =\frac{(-1)^{1 / 2 l} l !}{(1 / 2 l) !} \Psi(\xi, \eta) & & \text { for } l \text { even, } \\
& =0 & & \text { for } l \text { odd, }
\end{aligned}
$$

where $|\xi|<1$, and

$$
\Psi(\xi, \eta)=\left(1-\xi^{2}\right)^{-\mu-1 / 2 l} \sum_{k=0}^{\infty} \frac{(\mu)_{1 / 2 l+k}\left\{a_{k}\right\}}{k !}\left\{\frac{\eta^{2} \xi^{2}}{1-\xi^{2}}\right\}^{k},
$$

$A_{n}(\eta)$ is defined by (3.5) and $C_{n+l}^{\mu}(\xi)$ is the Gegenbauer polynomial.

(3.6), (3.9), and (3.12) are convergent; and $A_{n}(\eta), B_{n+l}(\eta), D_{n}(\eta)$ are assumed to exist.

Proof of (3.4). Putting $t=y / x$ in (1.3),

$$
\sum_{n=0}^{\infty} \frac{(y / x)^{n}}{n ! 2^{n}} H_{n}(x) H_{n+l}(y)=\frac{\exp \left(y^{2}\right)}{\left(1-y^{2} / x^{2}\right)^{1 / 2 l+1 / 2}} H_{l}(0) .
$$


Note that $H_{l}(0)=\epsilon$ where

$$
\epsilon=\frac{(-1)^{1 / 2 l} l !}{(1 / 2 l) !} \text { if } l \text { is even and } \epsilon=0 \text { if } l \text { is odd. }
$$

Using

$$
H_{n}(x)=\frac{(2 x)^{n}}{n !}{ }_{2} F_{0}\left[-1 / 2 n,-1 / 2 n+1 / 2 ;-;-1 / x^{2}\right] \text {, }
$$

and a corresponding expression for $H_{n+l}(y)$, putting $x=1 / z^{1 / 2}$, and expanding

$$
\left(1-y^{2} / x^{2}\right)^{-1 / 2 l-1 / 2}={ }_{1} F_{0}\left[1 / 2 l+1 / 2 ;-; z y^{2}\right]
$$

reduces (3.13) to

$$
\begin{gathered}
\sum_{n=0}^{\infty} \frac{2^{n+l} y^{2 n+l}}{n !}{ }_{2} F_{0}[-1 / 2 n,-1 / 2 n+1 / 2 ;-;-z] \\
\cdot{ }_{2} F_{0}\left[-1 / 2(n+l),-1 / 2(n+l)+1 / 2 ;-;-1 / y^{2}\right] \\
=\epsilon \exp \left(y^{2}\right)_{1} F_{0}\left[1 / 2 l+1 / 2 ;-; z y^{2}\right] .
\end{gathered}
$$

We take the inverse Laplace of (3.14) with respect to $z$ to give

$$
\begin{aligned}
\sum_{n=0}^{\infty} \frac{2^{n+l} y^{2 n+l}}{n !} & { }_{2} F_{0}\left[-1 / 2(n+l),-1 / 2(n+l)+1 / 2 ;-;-1 / y^{2}\right] \\
& \cdot{ }_{2} F_{1}\left[\begin{array}{cc}
-1 / 2 n,-1 / 2 n+1 / 2 ; & -z \\
1 / 2 l+1 / 2 ; &
\end{array}\right] \\
= & \epsilon \exp \left\{y^{2}(1+z)\right\} \\
= & \epsilon \exp \left(y^{2}\right) \sum_{k=0}^{\infty} \frac{\left(z y^{2}\right)^{k}}{k !} .
\end{aligned}
$$

Taking Laplace and inverse Laplace transforms of (3.15) and (3.17) with respect to $z$, and changing variables to $\xi$ and $\eta$, results in Eq. (3.4).

Proof of (3.7). Now (3.16) may also be expanded in the form

$$
\epsilon \sum_{k=0}^{\infty} \frac{(1+z)^{k} y^{2 k}}{k !}
$$

Taking transforms of (3.15). and (3.18) with respect to $y$ and $z$, and changing variables gives (3.7). The representations for the Gegenbauer polynomial

$$
\begin{aligned}
C_{m}^{\lambda}(x) & =\frac{(2 \lambda)_{m} x^{m}}{m !}{ }_{2} F_{1}\left[\begin{array}{cc}
-1 / 2 m,-1 / 2 m+1 / 2 ; & \frac{x^{2}-1}{x^{2}}
\end{array}\right] \\
\lambda+1 / 2 ; & \frac{(\lambda)_{m}(2 x)^{m}}{m !}{ }_{2} F_{1}\left[\begin{array}{cc}
-1 / 2 m,-1 / 2 m+1 / 2 ; & 1 / x^{2} \\
1-\lambda-m ; &
\end{array}\right]
\end{aligned}
$$

are used $[10, \mathrm{p} .1030]$.

Proof of (3.11). Taking Laplace transforms of (3.15) and (3.16) with respect to $y$, one has 


$$
\sum_{n=0}^{\infty} \frac{2^{n+l}(\mu+1 / 2 l)_{n+1 / 2 l} y^{2 n+l}}{n !}{ }_{2} F_{1}\left[\begin{array}{cc}
-1 / 2(n+l),-1 / 2(n+l)+1 / 2 & 1 / y^{2} \\
1-\mu-n-l ; &
\end{array}\right]
$$

$$
\begin{aligned}
& \cdot{ }_{2} F_{1}\left[\begin{array}{cc}
-1 / 2 n,-1 / 2 n+1 / 2 ; & -z \\
-1 / 2 l+1 / 2 ; &
\end{array}\right] \\
= & \epsilon\left\{1-y^{2}(1+z)\right\}^{-\mu-1 / 2 l} \\
= & \epsilon\left\{1-y^{2}\right\}^{-\mu-1 / 2 l} \sum_{k=0}^{\infty} \frac{\left(z y^{2}\right)^{k}(\mu+1 / 2 l)_{k}}{k !} .
\end{aligned}
$$

Taking transforms of (3.21) and (3.23) with respect to $z$ and changing variables gives (3.11). The representation (3.20) is employed.

The special case (3.1) may be deduced from (3.11) by applying the representation (3.19) to the polynomial $A_{n}(\eta)$. (3.2) is derived from (3.11), while (3.3) is derived from (3.4).

Department of Mathematics

California State University

Fresno, California 93740

1. R. ASKEY, Orthogonal Polynomials and Special Functions, Regional Conference Series in Applied Mathematics, no. 21, SIAM, Philadelphia, Pa., 1975.

2. H. BATEMAN, "A partial differential equation connected with the functions of the parabolic cylinder," Bull. Amer. Math. Soc., v. 41, 1935, pp. 884-893.

3. F. BRAFMAN, "Some generating functions for Laguerre and Hermite polynomials,"

Canad. J. Math., v. 9, 1957, pp. 180-187. MR 19, 28.

4. L. CARLITZ, "Some extensions of the Mehler formula," Collect. Math., v. 21, 1970, pp. 117-130. MR 43 \#5076.

5. M. E. COHEN, "On Jacobi functions and multiplication theorems for integrals of Bessel functions," J. Math. Anal. Appl. (In press.)

6. M. E. COHEN, "On expansion on problems: New classes of formulae for the classical functions," SIAM J. Math. Anal. (In press.)

7. A. ERDÉLYI, "Über eine Erzeugende Funktion von Produkten Hermitescher Polynome," Math. Z., v. 44, 1938, pp. 201-211.

8. E. FELDHEIM, "Développements en série de polynomes d'Hérmite et de Laguerre à l'aide des transformations de Gauss et de Hankel. I, II, III," Nederl. Akad. Wetensch Proc., v. 43, 1940, pp. 224-248, 378-389. MR 1, 232.

9. H. W. GOULD \& A. T. HOPPER, "Operational formulas connected with two generalizations of Hermite polynomials," Duke Math. J., v. 29, 1962, pp. 51-63. MR 24 \#A2689.

10. I. S. GRADŠTEĬN \& L. M. RYŽIK, Table of Integrals. Series, and Products, 4th ed., Fizmatgiz, Moscow, 1963; English transl., Academic Press, New York and London, 1965. MR 28 \#5198; 33 \#5952.

11. R. P. GUPTA \& G. C. JAIN, "A generalized Hermite distribution and its properties," SIAM J. Appl. Math., v. 27, 1974, pp. 359-363. MR 50 \#2580.

12. G. H. HARDY, "Summation of a series of polynomials of Laguerre," J. London Math. Soc., v. 7, 1932, pp. 138-139.

13. E. HILlE, “On Laguerre's series. II," Proc. Nat. Acad. Sci. U.S.A., v. 12, 1926, pp. 265269.

14. J. KAMPÉ de FÉRIET, Danske Vid. Selsk. Math. Fys. Medd., v. 5, 1923, no. 2.

15. E. LUKACS, Characteristic Functions, Griffin's Statistical Monographs \& Courses, no. 5, Hafner, New York, 1960. MR 23 \#A1392.

16. Y. L. LUKE, The Special Functions and Their Approximations. Vols. 1,2, Math. in Sci. and Engineering, vol. 53, Academic Press, New York and London, 1969. MR 39 \#3039; 40 \#2909. 
17. F. G. MEHLER, "Ueber die Entwichlung einer Funktion von beliebig vielen Variablen nach Laplaceschen Funktionen höhrer Ordnung," J. Reine Angew. Math., v. 66, 1866, pp. 161176.

18. O. V. SARMANOV \& Z. N. BRATOEVA, "Probabilistic properties of bilinear expansions of Hermite polynomials," Teor. Verojatnost. $i$ Primenen., v. 12, 1970, pp. 520-531 = Theor. Probability Appl., v. 12, 1970, pp. 470-481. MR 35 \#7372.

19. G. N. WATSON, "Notes on generating functions of polynomials: (2) Hermite polynomials," J. London Math. Soc., v. 8, 1933, pp. 194-199.

20. L. WEISNER, "Generating functions for Hermite functions," Canad. J. Math., v. 11, 1959, pp. 141-147. MR 22 \#786. 